\title{
Total isovolumic time, a marker of left ventricular global dyssynchrony, is prolonged with age
}

\author{
Gani Bajraktari*, Per Lindqvist, Michael Y. Henein \\ Public Health and Clinical Medicine, Umeå University, Umeå, Sweden
}

Background and Aim: Total isovolumic time (T-IVT) and Tei index both reflect global left ventricular (LV) global dyssynchrony. They have been shown to be sensitive in responding to myocardial revascularization and in predicting clinical outcome in heart failure patients. Since most of these patients are senior, determining the exact effect of age on such parameters remains mandatory. The aim of this study was to investigate the effect of age on LV T-IVT and Tei index compared with conventional systolic and diastolic parameters in a group of normal individuals.

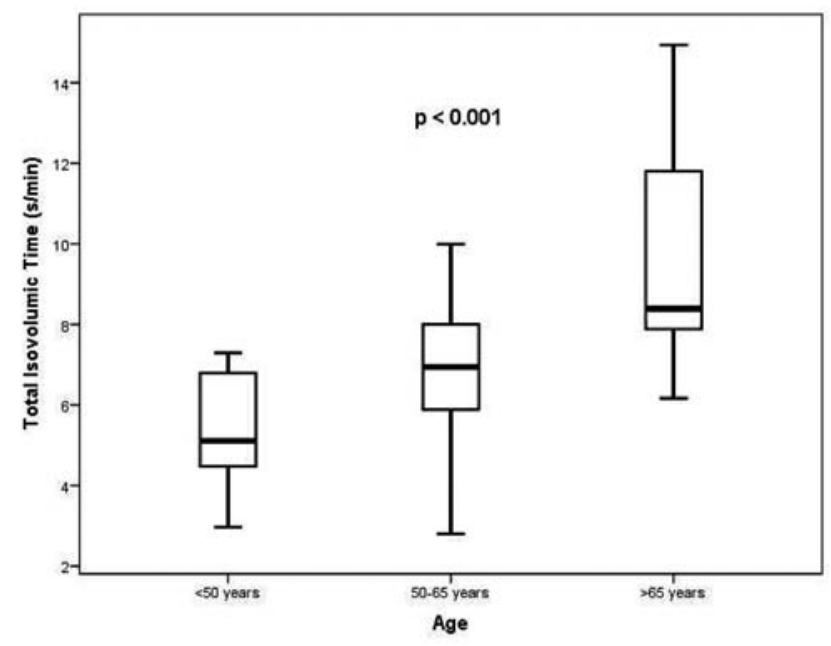

Figure 1. The pattern of change in Total Isovolumic Time with increasing age $(p<0.001)$.
Methods: We studied 47 healthy subjects, mean age 62 \pm 12 years, 23 males using conventional spectral Doppler echocardiography and tissue-Doppler imaging. T-IVT was measured as [ 60 - (total ejection time + total filling time) in $\mathrm{s} / \mathrm{min}]$, and Tei index calculated as (T-IVT/ejection time).

Results: LV ejection fraction was $68 \pm 6 \%$, E/A ratio $1 \pm 0.4$, filling time $538 \pm 136 \mathrm{~ms}$, ejection time $313 \pm 26 \mathrm{~ms}$, T-IVT $7.7 \pm 2.6 \mathrm{~s} / \mathrm{min}$ and Tei index $0.41 \pm 0.14$. Age strongly correlated with T-IVT $(r=0.8, p<0.001)$ and Tei index $(r=0.7$, $p<0.001)$. Age also correlated, although to a lesser extent, with $E / A$ ratio $(r=-0.6, p<0.001)$, but not bearing any relationship with global or segmental systolic function measurements.

Conclusions: In normal individuals, age is associated with exaggerated LV global dyssynchrony and diastolic function disturbances, but systolic function remains unaffected.

KEYWORDS: total isovolumic time, age, left ventricular dyssynchrony, Doppler echocardiography.

\section{Received: $20^{\text {th }}$ Mar 2013}

*Address for correspondence: "Rrethi i Spitalit", p.n., 10000 Prishtina, Kosovo.

Phone: +38138500600

E-mail: gani.bajraktari@medicin.umu.se

\section{Literature}

1. Duncan A, Francis D, Gibson D, Pepper J, Henein M. Electromechanical left ventricular resynchronisation by coronary artery bypass surgery. Eur J Cardiothorac Surg. 2004;26(4):711-9.

2. Bajraktari G, Duncan A, Pepper J, Henein M. Prolonged total isovolumic time predicts cardiac events following coronary artery bypass surgery. Eur $\mathrm{J}$ Echocardiogr. 2008;9(6):779-83.

3. Duncan AM, Francis DP, Henein MY, Gibson DG. Importance of left ventricular activation in determining myocardial performance (Tei) index: comparison with total isovolumic time. Int J Cardiol. 2004;95:211-7.

4. Tei C, Ling LH, Hodge DO, Bailey KR, Oh JK, Rodeheffer RJ, et al. New index of combined systolic and diastolic myocardial performance: a simple and reproducible measure of cardiac function - a study in normals and dilated cardiomyopathy. J Cardiol. 1995;26: 357-66. 